\title{
RESEARCH
}

Open Access

\section{Measuring patient-reported physical functioning and fatigue in myelodysplastic syndromes using a modular approach based on EORTC QLQ-C30}

Antoine Regnault ${ }^{1 *}$ (D, Farrah Pompilus ${ }^{2}$, Anna Ciesluk², Flora Mazerolle ${ }^{1}$, Rafael Bejar ${ }^{3}$, Robert J. Fram4, Douglas V. Faller ${ }^{4}$, Patrick Marquis ${ }^{2}$ and Jill A. Bell ${ }^{4}$

\begin{abstract}
Purpose: Physical functioning and fatigue are key patient concerns in myelodysplastic syndromes (MDS), chronic myelomonocytic leukemia (CMML), and acute myeloid leukemia (AML). The objective of this research was to generate supportive quantitative evidence for modular physical functioning and fatigue measures based on the European Organisation for Research and Treatment of Cancer (EORTC) Quality of Life Questionnaire Core 30 items (QLQ-C30) and a customized selection of 10 supplemental items from the EORTC Item Library.
\end{abstract}

Methods: The 40 items were completed online cross-sectionally by 51 patients (higher risk [HR] MDS: 53\%; CMML: 26\%; AML: 10\%). Psychometric analyses based on Rasch measurement theory (RMT) were conducted on the QLQC30 physical functioning and fatigue domains as well as measures combining QLQ-C30 and supplemental items. A measure of anemia-related symptoms composed of QLQ-C30 and supplemental items covering fatigue, dyspnea, and dizziness was also investigated.

Results: The QLQ-C30 physical functioning and fatigue domains showed good targeting to the sample and adequate reliability, with few conceptual gaps identified. Combining the QLQ-C30 and supplemental physical functioning and fatigue items improved the conceptual coverage and the reliability of the measures. The patientreported anemia-related symptom measure showed good measurement performance, underpinned by a clinically meaningful characterization of severity of these symptoms over a spectrum, starting with fatigue, then dyspnea, and finally dizziness (most severe).

Conclusion: The modular measurement approach of combining EORTC QLQ-C30 and Item Library offers a promising pragmatic solution to the measurement of physical functioning and fatigue, as well as anemia-related symptoms in clinical trials conducted in HR MDS, CMML, and AML.

Keywords: Patient-reported outcomes, Myelodysplastic syndromes, Acute myeloid leukemia, Chronic myelomonocytic leukemia, Fatigue, EORTC item library, Modular measurement approach, Physical functioning

\footnotetext{
* Correspondence: antoine,regnault@modusoutcomes.com

${ }^{1}$ Modus Outcomes, 4 Place Amédée Bonnet, 69002 Lyon, France

Full list of author information is available at the end of the article
}

\section{Springer Open}

(c) The Author(s). 2021 Open Access This article is licensed under a Creative Commons Attribution 4.0 International License, which permits use, sharing, adaptation, distribution and reproduction in any medium or format, as long as you give appropriate credit to the original author(s) and the source, provide a link to the Creative Commons licence, and indicate if changes were made. The images or other third party material in this article are included in the article's Creative Commons licence, unless indicated otherwise in a credit line to the material. If material is not included in the article's Creative Commons licence and your intended use is not permitted by statutory regulation or exceeds the permitted use, you will need to obtain permission directly from the copyright holder. To view a copy of this licence, visit http://creativecommons.org/licenses/by/4.0/. 


\section{Introduction}

Myelodysplastic syndromes (MDS), chronic myelomonocytic leukemia (CMML), and acute myeloid leukemia (AML) are rare blood cancers that affect the myeloid cells. They are associated with anemia, neutropenia, and thrombocytopenia, which lead to a variety of symptoms and functional impacts for affected patients [1]. Measuring patient-reported outcomes (PROs) is key to understanding the patient experience in the field of hematology in both research and practice $[2,3]$. Three core PRO concepts have been identified for oncology trials and should be measured to integrate the experience of patients and to demonstrate the benefit of new treatments as well as inform decision making: physical functioning, disease-related symptoms, and symptomatic adverse events [4]. The measurement of these core PRO concepts in oncology trials is increasingly performed using a modular approach. In a modular measurement approach, only the key concepts for a specific context of use are carefully selected and measured in the study, using items that are thoughtfully selected from existing static questionnaires and item banks or libraries $[4,5]$. This approach allows a bespoke and efficient measurement of the concepts that are meaningful to patients.

Assessing symptomatic adverse events of a new treatment for MDS, CMML, and AML will be dependent on the treatment under consideration, as different treatments may have different safety profiles. Patient reporting of symptomatic adverse events would typically be done using the PRO-Common Terminology Criteria for Adverse Events (PRO-CTCAE) [6, 7], with a selection of PRO-CTCAE items defined on a case-by-case basis depending on the expected adverse event symptom profile of each treatment [8].

In contrast, a measurement strategy for physical functioning and disease-related symptoms in MDS, CMML, and AML can be achieved independently of the treatment under consideration. For this purpose, one must identify the specific, key concepts to measure and the most appropriate instrument to use to assess these symptoms and aspects of physical functioning in this context of use. Previous research identified the key concepts of importance for patients and organized them into a conceptual model for the measurement of PROs in higher risk (HR) MDS, CMML, and AML [9]. Fatigue was identified as a core symptom, as well as other anemia-related symptoms, such as dyspnea and dizziness.

A modular approach based on the European Organisation for Research and Treatment of Cancer (EORTC) Quality of Life Questionnaire-Core 30 items (QLQ-C30) and Item Library is most appropriate to measure physical functioning and core symptoms of HR MDS, CMMT and AML [9]. The EORTC QLQ-C30 alone already includes domain scores for physical functioning and core symptoms of HR MDS, CMML, and AML, such as fatigue, and has shown good measurement performance for this population [10]. A modular approach combining the QLQ-C30 and a customized selection of items from the EORTC Item Library allows bridging the conceptual gaps identified on the QLQ-C30 compared to the framework previously created for HR MDS, CMML, and AML. Ten items from the EORTC Item Library were identified as potential supplementary items to the QLQ-C30 to create a customized measure of key PROs to capture clinical benefit in HR MDS, CMML, and AML for inclusion in clinical trials. This modular approach also has the advantage of offering a pragmatic solution to the challenges associated with the measurement of PROs in rare diseases [11], such as MDS, AML, or CMML, as it builds on existing established material and does not require researchers to develop new, disease-specific PRO measures.

The objective of this research was to generate supportive quantitative evidence on the appropriateness of using the EORTC QLQ-C30 and 10 supplemental items from the EORTC Item Library to assess key PROs for people with HR MDS, CMML, and AML; specifically, physical functioning, fatigue, and other anemia-related symptoms.

\section{Methods}

The items of the EORTC QLQ-C30 and 10 supplemental items chosen from the EORTC Item Library were compiled into an online survey. The survey was administered to people living with HR MDS, CMML, and AML at one time point.

\section{Patient sample}

Patients were recruited using convenience sampling (non-probability sampling) through the MDS Foundation, social media, and market research agencies. Patients were included if they met all of the following criteria: were willing to consent; spoke, read, and understood English; were located in the US at the time of survey completion; were 18 years old or older; had a selfreported diagnosis of HR MDS, CMML, or AML; and had a patient-rated Eastern Cooperative Oncology Group (ECOG) status of 0,1 , or 2 [12]. Patients were excluded if they met any of the following criteria: were receiving daunorubicin or idarubicin; had participated in a clinical trial for treatments related to MDS, CMML, or AML within the previous 2 weeks; underwent major surgery within the previous 2 weeks; had a diagnosis or had been treated for another cancer in the previous 2 years; had a life-threatening illness unrelated to cancer; had a visual, cognitive, or linguistic impairment preventing them from understanding and answering the survey questions; or had a patient-rated ECOG status of 3 or 4 . 
Recruitment was stopped when a total of 50 participants completing the online survey was reached. Given the practical challenges of recruitment in a rare disease context, a sample of 50 patients was deemed sufficient to generate early evidence on the item sets under scrutiny using the anticipated methods, especially as the items included in the analyses were carefully selected to be appropriate to the context of use and were therefore expected to be well targeted to the sample.

\section{Ethics}

All study documents and procedures were approved by an independent institutional review board (Copernicus Group IRB).

\section{EORTC QLQ-C30 and item library}

The EORTC QLQ-C30 questionnaire is a standard PRO instrument in cancer patient populations [13]. It has five functional scales, eight symptom scales, a finance item, and a global health and Quality of Life (QoL) scale (Table 1). All items have four response choices, except the global health QoL scale, which has seven. QLQ-C30 scale scores range from 0 to 100 . Higher scores represent better functioning/health status for the functioning scales and the global health status/QoL, but more severe symptoms for the symptom scales.

The EORTC Item Library is an online platform that compiles more than 900 individual items from over 60 EORTC questionnaires [14]. Users can select ad-hoc sets of items that were developed with the same general

Table 1 Number of EORTC QLQ-C30 and supplemental items from the EORTC Item Library to cover key patient-relevant concepts in HR MDS, CMML, and AML

\begin{tabular}{lll}
\hline & EORTC QLQ-C30 & EORTC Item Library \\
\hline Global health status/QoL (QL) & 2 & 2 \\
Physical functioning (PF) & 5 & 2 \\
Role functioning (RF) & 2 & \\
Emotional functioning (EF) & 4 & \\
Social functioning (SF) & 2 & 3 \\
Cognitive functioning (CF) & 2 & \\
Fatigue (FA) & 3 & 2 \\
Pain (PA) & 2 & \\
Dyspnea (DY) & 1 & \\
NauseaNomiting (NV) & 2 & \\
Insomnia (SL) & 1 & \\
Appetite loss (AP) & 1 & \\
Constipation (CO) & 1 & \\
Diarrhea (DI) & 1 & \\
Financial difficulties (FI) & 1 & \\
Dizziness (DZ) & & \\
\hline
\end{tabular}

principles as the EORTC QLQ-C30; items have the same response options and recall period as the QLQ-C30 and, for some items, translations are already available in a number of languages.

In our research, a set of 10 items from the EORTC Item Library were identified based on the conceptual model resulting from previous qualitative research and testing [9] (Table 1). These items were selected as they were measuring concepts strongly endorsed by patients or considered a core symptom/impact by clinicians, were not primarily considered a side-effect of treatment, and could potentially capture a treatment benefit. These 10 items were shown to be clear and relevant in cognitive debriefing interviews with patients with HR MDS, CMML, or AML. Of note, in addition to the items added to measure physical functioning (Difficulty climbing stairs, Feeling slowed down), fatigue (Weakness in arms or legs, Becoming easily tired, Lacking energy), and other anemia-related symptoms (Shortness of breath on exertion, Having to stop for breath when walking and Dizziness), two items assessing role functioning were also selected to be tested as complementary to the original QLQ-C30 role functioning (RF) domain.

\section{Statistical analyses}

Responses to the EORTC QLQ-C30 and 10 supplemental items from the EORTC Item Library were described. Psychometric analyses were performed in the Rasch measurement theory (RMT) framework. RMT uses a mathematical model, the Rasch model, to examine the legitimacy of creating scores from a set of items [15-17]. The following properties were explored in the RMT framework:

- Scale-to-sample targeting was assessed by visual inspection of the relative distribution of item locations and person measurements on their shared continuum.

- Adequacy of response scales was assessed by examining whether all 'item thresholds' (i.e., the point in the continuum where the most probable response between two adjacent response category changes) were properly ordered.

- Item fit was assessed by joint examination of statistical parameters (log residuals and chi-square values comparing between observed and expected responses to an item) and item characteristic curves (ICC) that graphically display the expected responses across the continuum of person scores and the observed values for each class interval of person scores. Fit residuals outside the recommended range of 2.5 / 2.5 and statistically significant chi-square values are indicative of possible fit issues [17]. 
- Reliability was assessed using Person Separation Index (PSI). Reliability coefficients are interpreted as follows: < 0.70: Poor; 0.70-0.79: Modest; 0.80-0.89: Adequate; 0.90-0.94: Good; > 0.95: Excellent [18].

- Local item dependency was assessed by examining the correlations between the standardized item residuals. Any positive correlation greater than 0.3 was considered as possibly indicative of item dependency [17].

Two sets of items for physical functioning and fatigue were tested separately using the Rasch model: first, items from the EORTC QLQ-C30 PF and FA domains and then the combination of the same QLQ-C30 items with the supplementary items from the EORTC Item Library. All items reflecting patient-reported anemia-related symptoms (i.e. items pertaining to fatigue, dyspnea, and dizziness) were also tested together in a separate Rasch model. The Rasch model was also applied to the items pertaining to role functioning but the results of this analyses are not presented here.

The RMT analyses were performed using RUMM2030 (RUMM Laboratory; Perth, Australia). All other statistical analyses were performed using SAS v9.4 (SAS Institute; Cary, NC, USA).

\section{Results}

\section{Patient demographics}

A total of 51 patients participated in the online survey, with most patients diagnosed with HR MDS (HR MDS: 53\%; CMML: 26\%; AML: 10\%). All patients confirmed a diagnosis of HR MDS, CMML, and AML to be included in the study. However, 6 patients did not report which was their exact diagnosis. The mean age was 68 years (SD: 12) and $49 \%$ of patients were female (Table 2).

\section{Description of responses to EORTC QLQ-C30 and item library items}

A possible floor effect for several EORTC QLQ-C30 items was identified (i.e., a substantial percentage of responders reported the lowest value ['Not at all']), whereas the responses to the supplemental items were well distributed across the response categories, with the exception of "dizziness" for which a greater number of patients responded 'Not at all' (Fig. 1). This confirmed that the customized supplemental item selection was well targeted to the patient sample overall.

\section{RMT analysis of physical functioning items}

The EORTC QLQ-C30 PF items demonstrated acceptable measurement performance: good targeting of the items to the patient sample (Fig. 2), adequate reliability, fit of all items to the Rasch model, and intended function of response scales for all items but one (hygiene-
Table 2 Sample description

\begin{tabular}{|c|c|}
\hline & $\begin{array}{l}\text { Total } \\
(N=51)\end{array}$ \\
\hline \multicolumn{2}{|l|}{ Age (in years) } \\
\hline Mean (SD) & $68(12)$ \\
\hline Median (Min - Max) & $71(26-84)$ \\
\hline Gender - Female N (\%) & $25(49)$ \\
\hline \multicolumn{2}{|l|}{ Employment status - N (\%) } \\
\hline Working full-time & $7(14)$ \\
\hline Working part-time & $4(8)$ \\
\hline Retired & $22(43)$ \\
\hline Other* & $12(24)$ \\
\hline Missing & $6(12)$ \\
\hline \multicolumn{2}{|l|}{ Living situation } \\
\hline Lives alone & $12(24)$ \\
\hline Lives in a nursing home or assisted living facility & $1(2)$ \\
\hline Lives with a partner, spouse, family or friends & $32(63)$ \\
\hline Missing & $6(12)$ \\
\hline \multicolumn{2}{|l|}{ Diagnosis - N (\%) } \\
\hline MDS & $27(53)$ \\
\hline CMML & $13(26)$ \\
\hline AML & $5(10)$ \\
\hline Missing** & $6(12)$ \\
\hline \multicolumn{2}{|l|}{ Number of years since diagnosis) } \\
\hline Mean (SD) & $5.0(7.4)$ \\
\hline Median (Min - Max) & $3(0-46)$ \\
\hline \multicolumn{2}{|c|}{ Prognostic risk category (IPSS-R) $)^{* * *}-\mathrm{N}(\%)$ in MDS only (Total $\left.N=27\right)$} \\
\hline Very high (> 6 points) & $1(4)$ \\
\hline High (> 4.5-6 points) & $10(37)$ \\
\hline Intermediate (> 3-4.5 points) & $3(11)$ \\
\hline Don't know & $13(48)$ \\
\hline \multicolumn{2}{|l|}{ ECOG status - N (\%) } \\
\hline 0 - Fully active & $7(14)$ \\
\hline $\begin{array}{l}1 \text { - Restricted in physically strenuous activity } \\
\text { but ambulatory }\end{array}$ & $12(24)$ \\
\hline 2 - Ambulatory and capable of all selfcare & $4(9)$ \\
\hline Missing & $28(55)$ \\
\hline Stem cell transplant - N (\%) & $1(2)$ \\
\hline Patients using supportive care therapies $* * * *$ & $18(35)$ \\
\hline
\end{tabular}

*Other employment status included student, not employed, retired-not employed, and disabled-not employed

**Missing diagnosis: patient with MDS, CMML, or AML who did not complete the question on the demographic and health information form

***Prognostic risk category only assessed for patients with HR MDS and CMML $(n=40)$

****Examples of supportive care therapies include transfusions, granulocyte colony stimulating factor, granulocyte macrophage-colony stimulating factor, thrombopoietin, erythropoiesis stimulating agents, etc. 


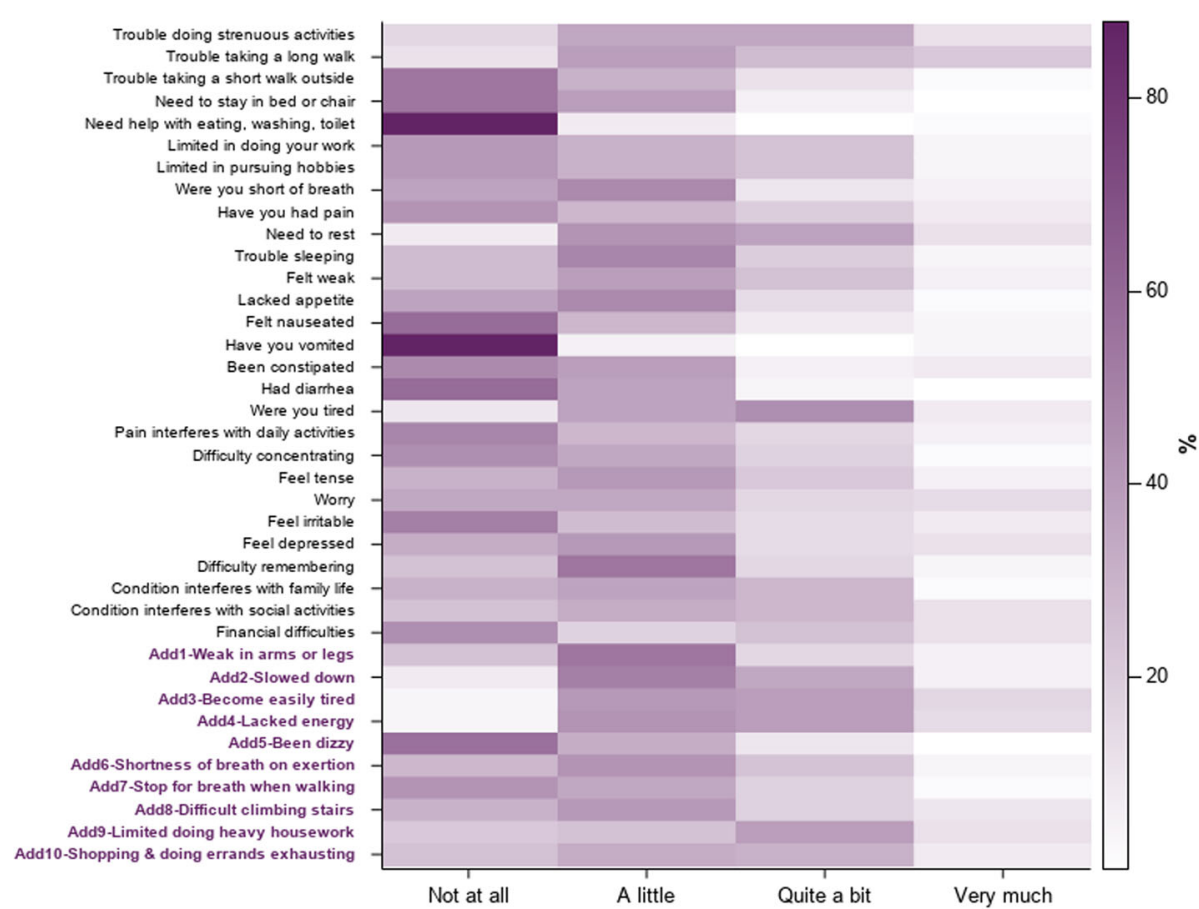

Fig. 1 Distribution of the responses to the original EORTC QLQ-C30 items and supplemental items from the EORTC Item Library ( $N=51)$. Darker colors indicate higher percentages of patients who endorsed the response. Items with bold purple labels are the 10 supplemental items from the EORTC Item Library

related) (Table 3 and Supplementary material 1 and 2). No pairs of items showed high correlations in standardized residuals (see supplementary material 1 ). Some gaps in the coverage of the physical functioning continuum were uncovered; for example, few items provided an opportunity to differentiate between scores of patients with higher levels of physical functioning (Table 3).
The addition of the two physical functioning items from the EORTC Item Library to the QLQ-C30 PF items bridged some gaps in the coverage of the functioning continuum, especially thresholds between 0 and 1 logit and between 2 and 4 logits (where no threshold was originally present) and at about 5 logits (Fig. 2). The new items therefore added information for a part of the physical functioning continuum where most patients

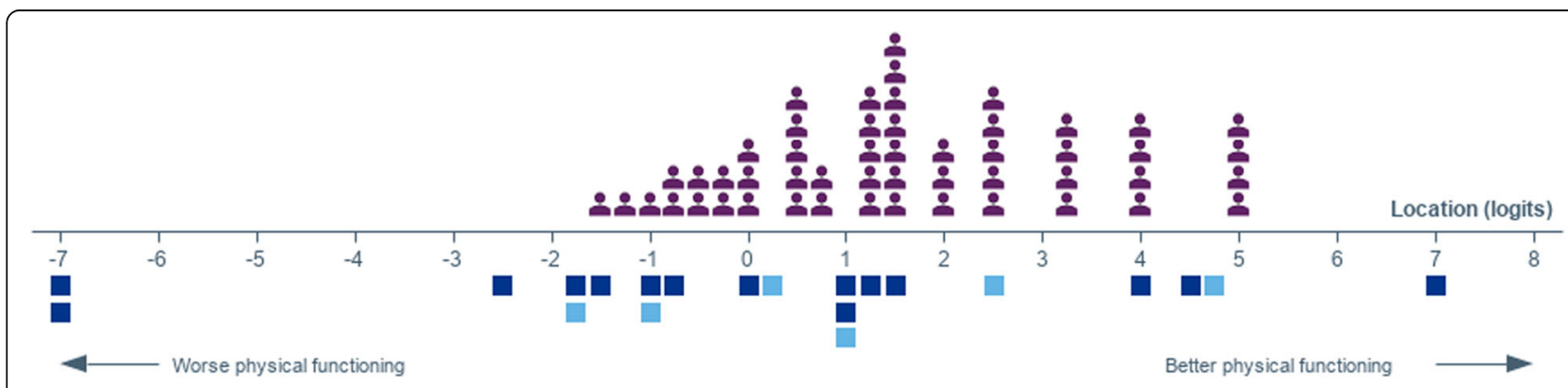

Physical functioning + $\square$ EORTC QLQ-C30 items $\square$ EORTC supplemental items

Fig. 2 Scale to sample targeting of the original EORTC QLQ-C30 and supplemental Physical Functioning (PF) items (N = 51). The upper panel shows the distribution of the 51 individuals of the survey sample over the physical functioning continuum; the lower panel (blue squares) show the distribution of the 'response thresholds' (i.e., the point of the continuum where the most probable response between two adjacent response categories for an item changes) of the EORTC QLQ-C30 and supplemental physical functioning items on the physical functioning continuum 
Table 3 Summary results of the Rasch measurement theory analysis of 1) the EORTC QLQ-C30 PF items and 2) EORTC QLQ-C30 and supplemental PF items

\begin{tabular}{|c|c|c|c|c|c|c|}
\hline & \multicolumn{3}{|c|}{ EORTC QLQ-C30 Physical functioning } & \multicolumn{3}{|c|}{ EORTC QLQ-C30 Physical functioning and supplemental items } \\
\hline & Disordered threshold & Fit residuals & PSI & Disordered threshold & Fit residuals & PSI \\
\hline Trouble doing strenuous activities & No & -0.771 & 0.82 & No & -0.939 & 0.83 \\
\hline Trouble taking a long walk & No & -1.767 & & No & -1.506 & \\
\hline Trouble doing short walk out & No & -0.692 & & No & -0.766 & \\
\hline Need to stay in bed or chair & No & 2.334 & & No & 1.841 & \\
\hline Need help with eat wash toilet & Yes & -0.93 & & Yes & -0.829 & \\
\hline Slowed down & & & & No & 0.795 & \\
\hline Difficult climbing stairs & & & & No & -0.291 & \\
\hline
\end{tabular}

where distributed. It also marginally improved reliability, without adding any issue in terms of fit, item dependency, or adequacy of the response options (Table 3 and Supplementary material 1).

\section{RMT analysis of fatigue and other anemia-related symptoms}

The EORTC QLQ-C30 FA items demonstrated acceptable measurement performance: good targeting of the items to the patient sample (Fig. 3), adequate reliability, fit of all items to the Rasch model, and intended functioning of all response scales (Table 4 and Supplementary material 1 and 2). No pairs of items showed high correlations in standardized residuals (see supplementary material 1). However, some gaps in the coverage of the fatigue continuum were uncovered (Fig. 3).

The addition of the three fatigue items from the EORTC Item Library to the QLQ-C30 FA items bridged some gaps in the coverage of the fatigue continuum (Table 4). It also improved reliability to a very good level, without adding any issue in terms of fit, or adequacy of the response options (Table 4). The items "Becoming easily tired" and "Lacking energy" showed some possible dependency (Standardized residual correlation: 0.45 - Supplementary material 1).

Combining the items assessing fatigue, dyspnea, and dizziness in a single scale intended to measure the severity of symptoms related to anemia among patients with HR MDS, CMML, and AML led to a measure with adequate measurement performances: good targeting (data not shown), good reliability, fit of all items to the Rasch model, and no issues in terms of response scales. Some possible dependency was found between three pairs of items "Becoming easily tired" and "Lacking energy" (0.69); "Shortness of breath" and "Shortness of breath on exertion" (0.65); "Become easily tired" and "Weakness in arms or legs" (0.35) - Supplementary material 1). Most importantly, the RMT analysis uncovered a meaningful hierarchy of the anemia-related symptom items, in which fatigue comes first, followed by dyspnea, and dizziness (Fig. 4).

Proposed new scores specific to HR MDS, CMML and AML using EORTC QLQ-C30 and supplemental items

The RMT analyses warrant the creation of scores combining the items from the QLQ-C30 and Item Library

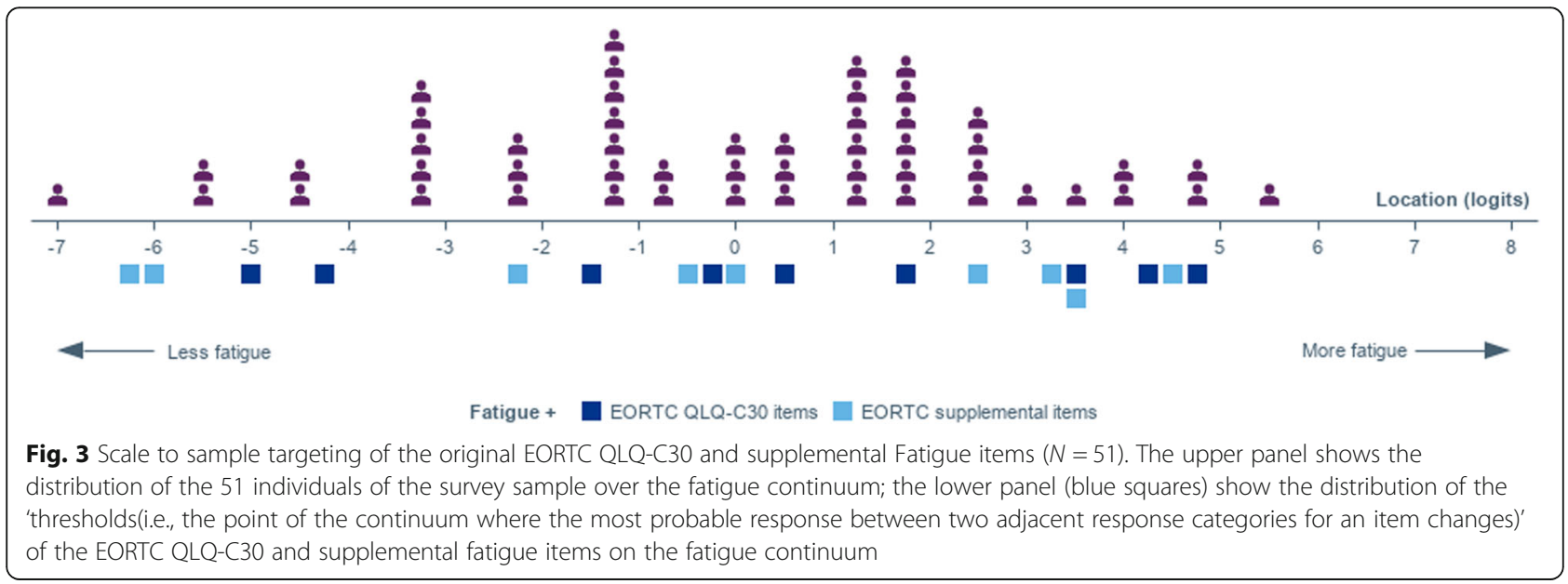


Table 4 Summary results of the Rasch measurement theory analysis of 1) the EORTC QLQ-C30 FA items, 2) the EORTC QLQ-C30 and supplemental FA items, and 3) the EORTC QLQ-C30 and supplemental items assessing fatigue and other anemia-related symptoms

\begin{tabular}{|c|c|c|c|c|c|c|c|c|c|}
\hline & \multicolumn{3}{|c|}{ EORTC QLQ-C30 Fatigue } & \multicolumn{3}{|c|}{$\begin{array}{l}\text { EORTC QLQ-C30 Fatigue and } \\
\text { supplemental items }\end{array}$} & \multicolumn{3}{|c|}{$\begin{array}{l}\text { Fatigue and other anemia-related } \\
\text { symptoms }\end{array}$} \\
\hline & $\begin{array}{l}\text { Disordered } \\
\text { threshold }\end{array}$ & Fit residuals & PSI & $\begin{array}{l}\text { Disordered } \\
\text { threshold }\end{array}$ & Fit residuals & PSI & $\begin{array}{l}\text { Disordered } \\
\text { threshold }\end{array}$ & Fit residuals & PSI \\
\hline Need to rest & No & -0.375 & 0.82 & No & 0.515 & 0.90 & No & -0.205 & 0.89 \\
\hline Felt weak & No & -0.171 & & No & 0.462 & & No & -0.021 & \\
\hline Were you tired & No & 0.242 & & No & 0.159 & & No & -0.760 & \\
\hline Weak in arms or legs & & & & No & 0.233 & & No & 0.244 & \\
\hline Become easily tired & & & & No & -2.091 & & No & -1.171 & \\
\hline Lacked energy & & & & No & -0.917 & & No & -0.506 & \\
\hline Were you short of breath & & & & & & & No & 0.777 & \\
\hline Shortness breath on exertion & & & & & & & No & 0.660 & \\
\hline Stop for breath when walking & & & & & & & No & 1.357 & \\
\hline Been dizzy & & & & & & & No & 0.777 & \\
\hline
\end{tabular}

for PF, FA, and a combination of all items assessing fatigue and other symptoms related to anemia. For consistency, we recommend that the calculation of these scores follow the same principles as the calculation of the QLQ-C30 scores, keeping a range from 0 to 100.

\section{Discussion}

In this research, we created customized PRO measures of physical functioning and fatigue for HR MDS, CMML, and AML using items from the EORTC QLQ-C30 and Item Library. We also explored a wider measure targeting the symptomatic manifestations of anemia, a core clinical feature of HR MDS, CMML, and AML, based on 10 PRO items covering fatigue plus dyspnea and dizziness. Quantitative evidence from the application of modern psychometrics in a sample of 51 patients supported the appropriateness of the customized measures in this context.

Our modular measurement approach to the key PROs in HR MDS, CMML, and AML was conceptually driven. We first conducted sound qualitative research including a literature review, interviews with clinicians and patients, and the development of a conceptual model

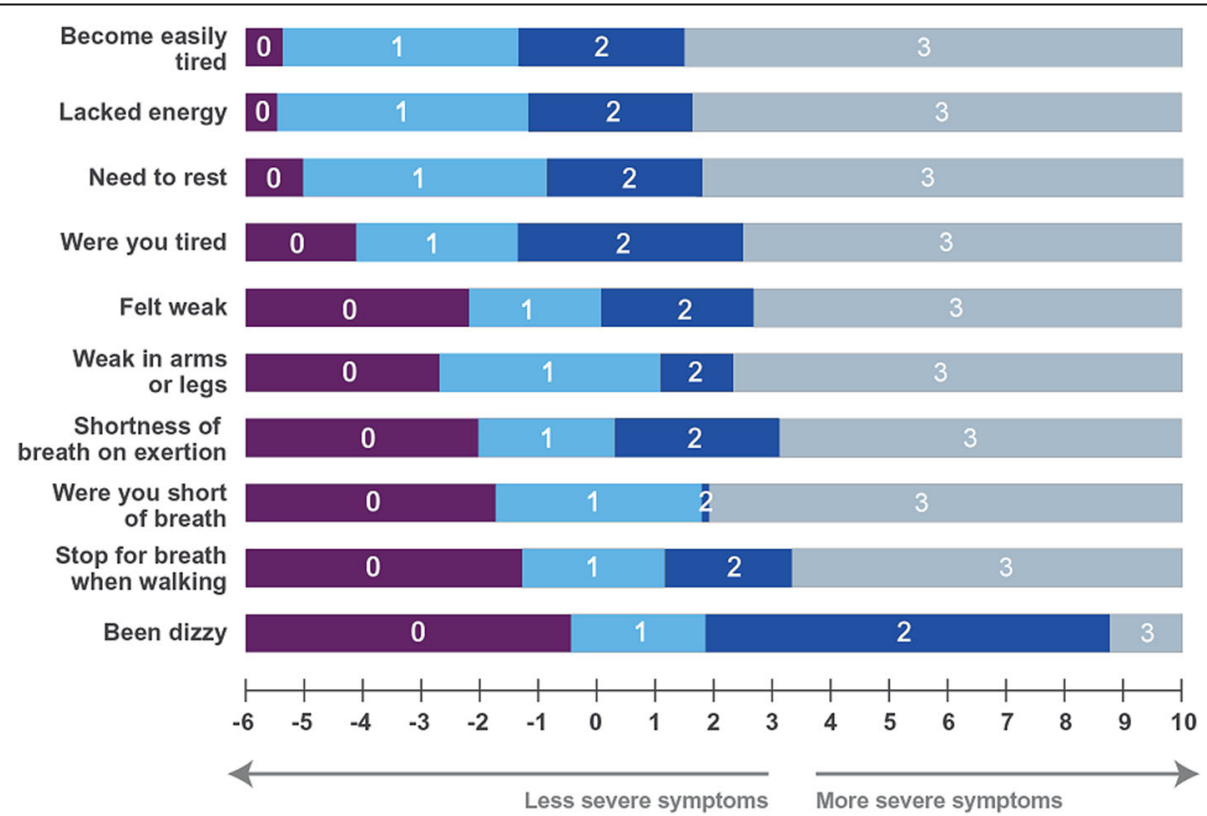

Fig. 4 The EORTC QLQ-C30 and Item Library items delineates the progression of anemia-related symptom severity from fatigue to dyspnea and dizziness in HR MDS, CMML, and AML patients. Item threshold map from RMT analysis on online survey data $(N=51)$ For each item, the most probable response is presented depending on the position on the symptom severity continuum (0: Not at all; 1: A little; 2: Quite a bit; 3: Very much) 
summarizing the key outcomes for patients with MDS, CMML, and AML [9]. We then used the EORTC Item Library to complement the QLQ-C30 based on the conceptual model. The analyses described here generated supportive evidence of the relevance of the modular measurement approach previously described and available by using the EORTC Item Library as a resource to supplement legacy EORTC instrument; our research show how this method ensures that PROs are well suited for specific contexts of use like rare cancers. We showed that the EORTC QLQ-C30 scores had acceptable performance in patients with HR MDS, CMML, and AML and that the supplemental items selected from the EORTC Item Library improved them. The conceptual clarity made possible by the EORTC QLQ-C30 and supplemental items is illustrated by the clinically meaningful ordering of anemia-related symptoms uncovered by the RMT analysis of these data: it showed that the perception of symptoms of anemia by patients with HR MDS, CMML and AML appears to start by fatigue (first fatigability, then weakness) then dyspnea (first on exercise, then at rest), and finally dizziness. The positive results of our RMT analyses for the various item sets that we investigated and the informative findings from our analyses is certainly stemming from the early thoughtful conceptualization and qualitative work conducted with patients to carefully craft the item sets that are used to measure the concepts of interest in the specific context of the research. Thus, we would recommend that any modular measurement research endeavor put emphasis on these initial stages.

Our research focused on the measurement of core outcomes for patients with HR MDS, CMML, or AML, namely physical functioning, fatigue, and anemia-related symptoms. Yet, other concepts are relevant to capture the full patient experience in this context. First, role functioning is recognized as an important PRO concept and was identified in our conceptual model. However, while we added two items from the EORTC Item Library based on our predefined conceptualization of patient experience with MDS, CMML, and AML to the two QLQC30 RF items, we were not able to demonstrate that the resulting item set had strong measurement properties. Including more role functioning items, for example from the 10-item bank used for the EORTC role functioning computer adaptive test [19], may be an option to create a measure of role functioning with better measurement properties based on the EORTC measurement system. Besides, PRO instruments such as the QOL-E [20] and the Quality of Life in Myelodysplasia Scale (QUALMS) [21] have been developed to measure more distal concepts, such as health-related quality of life, in MDS. The possibility that these instruments may be relevant to capture role functioning should be explored. Finally, patients with HR MDS, CMML, or AML also experience other symptoms related to cytopenia, for example related to thrombocytopenia or neutropenia. Further research on an acceptable measurement approach to these symptoms would allow a more comprehensive understanding of the experience of patients with HR MDS, CMML, or AML.

We also acknowledge some limitations in the data collected for our online study. Firstly, the sample consisted of only 51 subjects. The RMT parameters could be estimated in this relatively small sample, with a reasonable amount of uncertainty, but our results should be confirmed in larger samples. Typically, the specific examination of the measures in each diagnosis subgroup (HR MDS, CMML, and AML) needs to be further explored to confirm the absence of major differences in responses of patients from a different diagnosis subgroup. It also would be relevant to explore whether the measure of anemia-related symptoms allows characterizing the disease continuum from MDS to AML in a larger sample including enough patients within each subgroup. This may show, for example, that patients with AML are more likely to report more severe anemia-related symptoms, such as dizziness. Secondly, our sample was a convenience sample, and all the variables were reported by patients, including their diagnosis. This raises questions about how representative this sample is of the population of patient with HR MDS, CMML, and AML and the extent to which our findings in terms of coverage of the physical functioning and fatigue continuums by our item selection, as well as the good fit of the selected items to the Rasch model, are generalizable. Replicating our analyses in a larger, better clinically defined sample would reinforce our findings. For example, having a sample where the QLQ-C30 and supplemental items are collected together with good quality hemoglobin level data would allow for exploration of the association between hemoglobin level and severity of fatigue or anemia-related symptoms, hence consolidating their validity. Similarly, the construct validity of the scores based on the QLQ-C30 and supplemental items could be consolidated in future research by exploring their association with other measures of fatigue and physical functioning. Finally, the study was cross-sectional, preventing the examination of the longitudinal measurement properties of the PRO measures, such as test-retest reliability, ability to detect change over time, or exploration of what constitutes a meaningful change in score. Thus, more data will be needed to confirm our positive early results on the appropriateness of these modular measures of physical functioning and fatigue in this context.

\section{Conclusion}

The EORTC QLQ-C30 and the selection of items from the EORTC Item Library offer a promising pragmatic 
solution to measure physical functioning and key disease-related symptoms (fatigue and other anemiarelated symptoms) in HR MDS, CMML, and AML. Specifically, the QLQ-C30 plus supplemental items could contribute to the demonstration of the benefit of new treatments in the context of clinical trials in this patient population.

\section{Supplementary Information}

The online version contains supplementary material available at https://doi. org/10.1186/s41687-021-00334-w.

\section{Additional file 1}

Additional file 2. Supplemental materials: Item characteristic curves.

\section{Acknowledgments}

The authors wish to acknowledge and thank the patients who participated in the study. Support with the conduct of the study and preparation of the manuscript was provided by Alissa Rams.

This research was funded by Millennium Pharmaceuticals, Inc., Cambridge, MA, a wholly owned subsidiary of Takeda Pharmaceutical Company Limited.

\section{Authors' contributions}

AR designed and performed the analyses, participated to the design of the study, interpretation of the results, and preparation of the manuscript. FP, $A C$, and JB participated in the design of the study, data collection, interpretation of results and preparation of the manuscript. FM performed the analyses, participated in the interpretation of results and preparation of the manuscript. RB, RF, DF, and PM participated in the interpretation of results and preparation of the manuscript. All authors read and approved the final manuscript.

\section{Funding}

The study was funded by Millennium Pharmaceuticals, Inc., Cambridge, MA, a wholly owned subsidiary of Takeda Pharmaceutical Company Limited.

\section{Availability of data and materials}

For inquiries regarding the data set and possibilities of data sharing, please contact the authors.

\section{Declarations}

\section{Ethical approval and consent to participate}

All study documents and procedures were approved by an independent institutional review board. Patients provided written consent to participate.

\section{Consent for publication}

All patients provided written consent to have the results of this study published in medical journals. The consent form informed patients that no personal information will be revealed.

\section{Competing interests}

The study was funded by Millennium Pharmaceuticals, Inc., Cambridge, MA a wholly owned subsidiary of Takeda Pharmaceutical Company Limited. JB, RF and DF are employees of and have ownership interest in Takeda Pharmaceuticals. AR, FP, AC, FM and PM are employees of Modus Outcomes, which received payment from Takeda Pharmaceuticals to conduct this research. RB declares employment and equity with Aptose Biosciences.

\section{Author details}

${ }^{1}$ Modus Outcomes, 4 Place Amédée Bonnet, 69002 Lyon, France. ${ }^{2}$ Modus Outcomes, Cambridge, MA, USA. ${ }^{3}$ Moores Cancer Center, UC San Diego MDS Center of Excellence, San Diego, CA, USA. ${ }^{4}$ Millennium Pharmaceuticals, Inc., a wholly owned subsidiary of Takeda Pharmaceutical Company Limited, Cambridge, MA, USA.
Received: 13 November 2020 Accepted: 29 June 2021

Published online: 20 July 2021

\section{References}

1. Prebet, T., \& Zeidan, A. (2016). Trends in clinical investigation for myelodysplastic syndromes. Clin Lymphoma Myeloma Leuk, 16(Suppl), S57S63. https://doi.org/10.1016/j.clml.2016.02.012

2. Acquadro, C., \& Regnault, A. (2015). Patient-reported outcomes in drug development for hematology. Hematology Am Soc Hematol Educ Program, 2015(1), 496-500. https://doi.org/10.1182/asheducation-2015.1.496.

3. Efficace, F., Gaidano, G., \& Lo-Coco, F. (2017). Patient-reported outcomes in hematology: Is it time to focus more on them in clinical trials and hematology practice? Blood., 130(7), 859-866. https://doi.org/10.1182/ blood-2017-03-737403

4. Kluetz, P. G., Slagle, A., Papadopoulos, E. J., Johnson, L. L., Donoghue, M. Kwitkowski, V. E., ... Pazdur, R. (2016). Focusing on core patient-reported outcomes in cancer clinical trials: Symptomatic adverse events, physical function, and disease-related symptoms. Clin Cancer Res, 22(7), 1553-1558. https://doi.org/10.1158/1078-0432.CCR-15-2035.

5. Kluetz, P. G., Papadopoulos, E. J., Johnson, L. L., Donoghue, M., Kwitkowski, V. E., Chen, W. H., ... Pazdur, R. (2016). Focusing on Core patient-reported outcomes in Cancer clinical trials-response. Clin Cancer Res, 22(22), 5618. https://doi.org/10.1158/1078-0432.CCR-16-2140.

6. Kluetz, P. G., Chingos, D. T., Basch, E. M., \& Mitchell, S. A. (2016). Patientreported outcomes in Cancer clinical trials: Measuring symptomatic adverse events with the National Cancer Institute's patient-reported outcomes version of the common terminology criteria for adverse events (PRO-CTCA E). Am Soc Clin Oncol Educ Book, 35, 67-73. https://doi.org/10.1200/EDBK_1 59514.

7. Basch E, Reeve BB, Mitchell SA, et al. Development of the National Cancer Institute's patient-reported outcomes version of the common terminology criteria for adverse events (PRO-CTCAE). J Natl Cancer Inst. 2014;106(9).

8. Trask, P. C., Dueck, A. C., Piault, E., \& Campbell, A. (2018). Patient-reported outcomes version of the common terminology criteria for adverse events: Methods for item selection in industry-sponsored oncology clinical trials. Clin Trials, 15(6), 616-623. https://doi.org/10.1177/1740774518799985.

9. Bell, J. A., Galaznik, A., Pompilus, F., Strzok, S., Bejar, R., Scipione, F., Marquis, P. (2019). A pragmatic patient-reported outcome strategy for rare disease clinical trials: Application of the EORTC item library to myelodysplastic syndromes, chronic myelomonocytic leukemia, and acute myeloid leukemia. J Patient Rep Outcomes, 3(1), 35. https://doi.org/10.1186/ s41687-019-0123-4.

10. Sommer, K., Cottone, F., Aaronson, N. K., Fayers, P., Fazi, P., Rosti, G., .. Efficace, F. (2020). Consistency matters: Measurement invariance of the EORTC QLQ-C30 questionnaire in patients with hematologic malignancies. Qual Life Res, 29(3), 815-823. https://doi.org/10.1007/s11136-019-02369-5.

11. Morel, T., \& Cano, S. J. (2017). Measuring what matters to rare disease patients - reflections on the work by the IRDiRC taskforce on patientcentered outcome measures. Orphanet J Rare Dis, 12(1), 171. https://doi. org/10.1186/s13023-017-0718-x.

12. Oken, M. M., Creech, R. H., Tormey, D. C., Horton, J., Davis, T. E., McFadden, E. T., \& Carbone, P. P. (1982). Toxicity and response criteria of the eastern cooperative oncology group. Am J Clin Oncol, 5(6), 649-655. https://doi. org/10.1097/00000421-198212000-00014.

13. Aaronson, N. K. (1993). Assessment of quality of life and benefits from adjuvant therapies in breast cancer. Recent Results Cancer Res, 127, 201-210. https://doi.org/10.1007/978-3-642-84745-5 28.

14. Kuliś, D. B. A., Whittaker, C., van de Poll-Franse, L. V., Darlington, A., Holzner, B., Koller, M..... Grønvold, M. (2017). The Use of the EORTC Item Library to Supplement EORTC Quality of Life Instruments. Glasgow: The International Society for Pharmacoeconomics and Outcomes Research (ISPOR) 20th Annual European Congress.

15. Rasch, G. (1960). Probabilistic models for some intelligence and achievement tests. Copenhagen: Danish Institute for Educational Research (Expanded edition, 1980. Chicago: University of Chicago Press).

16. Andrich, D. (2011). Rating scales and Rasch measurement. Expert Rev Pharmacoecon Outcomes Res, 11(5), 571-585. https://doi.org/10.1586/ erp.11.59.

17. Hobart, J., \& Cano, S. (2009). Improving the evaluation of therapeutic interventions in multiple sclerosis: the role of new psychometric methods. Health Technol Assess, 13(12), iii, ix-x, 1-177. 
18. Nunnally, J. C. (1994). Psychometric theory (ed third edition): Tata McGraw-hill education.

19. Gamper, E. M., Petersen, M. A., Aaronson, N., et al. (2016). Development of an item bank for the EORTC role functioning computer adaptive test (EORTC RF-CAT). Health Qual Life Outcomes, 14(1), 72. https://doi.org/10.11 86/s12955-016-0475-x.

20. Oliva, E. N. D. B., D'Angelo, A., et al. (2001). QOL-E: A new tool for the assessment of quality of life (QOL) in myelodysplastic syndrome (MDS). Blood, 98, 427a.

21. Abel, G. A., Efficace, F., Buckstein, R. J., Tinsley, S., Jurcic, J. G., Martins, Y., .. Klaassen, R. J. (2016). Prospective international validation of the quality of life in myelodysplasia scale (QUALMS). Haematologica., 101(6), 781-788. https://doi.org/10.3324/haematol.2015.140335.

\section{Publisher's Note}

Springer Nature remains neutral with regard to jurisdictional claims in published maps and institutional affiliations.

\section{Submit your manuscript to a SpringerOpen ${ }^{\odot}$ journal and benefit from:}

- Convenient online submission

- Rigorous peer review

- Open access: articles freely available online

High visibility within the field

- Retaining the copyright to your article 